\title{
Herramientas teóricas para la definición y análisis de las "candidaturas independientes" locales: nuevas posibilidades de estudio en el ámbito municipal español
}

\author{
Miriam Ureta García \\ UNIVERSIDAD DEL PAÍS VASCO, LEIOA (BIZKAIA), ESPAÑA \\ miriam.ureta@ehu.eus
}

\begin{abstract}
Resumen: Esta investigación nace con vocación de ofrecer herramientas teóricas que sirvan para definir, clasificar y analizar el fenómeno de las "candidaturas independientes", con especial atención al caso español. Así, el primer objetivo de este trabajo es proponer un esquema de categorización integral que conecte las contribuciones de diferentes autores a la hora de clasificar en subtipologías realidades diversas derivadas de la categoría general de "candidaturas independientes". El segundo objetivo es presentar evidencias empíricas a partir de las cuales se fundamentan las nuevas posibilidades de estudio de "candidaturas independientes" en la esfera municipal española. Mientras que, finalmente, el tercer objetivo persigue conocer los factores estructurales generales a partir de los cuales se puede comenzar a explicar por qué surge y aumenta en votos la unidad de análisis de la investigación.
\end{abstract}

Palabras clave: candidaturas independientes, política local española, municipalismo

Abstract: This work aims to offer theoretical tools in order to define, classify and analyze the phenomenon of "independent candidacies", paying special attention to the Spanish case. Therefore, the first goal of this study is to propose a complete categorization scheme which connects different authors' contributions when it comes to classifying into subtipologies diverse realities derivative from the general category of "independent candidacies". The second objective is to present empirical evidence on which the new possibilities of the study of "independent candidacies" at the Spanish local level are based. Whereas, finally, the third goal seeks to know which are the general structural factors from which it can be explained why the unit of analysis of this research emerges and grows in terms of votes.

Keywords: independent candidacies, Spanish local politics, localism

1. Definiendo las "candidaturas independientes": hacia un modelo de clasificación integral

la hora de definir el concepto "candidaturas independientes" la literatura exis-
tente señala dos características que suponen un mínimo común denominador
de todas ellas: (i) no asumen las siglas de grandes partidos convencionales/ 
estatales y (ii) son una realidad que opera específica y exclusivamente a nivel local (Bracanti, 2008; Boogers, 2008; Holtmann, 2008; Copus et al., 2008, 2009; González, 2012; Ubasart, 2012; Reuchamps et al., 2013; Bautista 2015; Arengenedt, 2016; Heyerick, 2016; Maskarinec y Klimovsky, 2016; Vampa, 2016; Kening y Rahat, 2018). Así, el término "candidaturas independientes" ha venido a funcionar en la teoría de referencia como una categoría general, pero esta definición es insuficiente y se hace necesaria una mayor precisión a nivel conceptual. Es por ello que muchos de los autores arriba citados han profundizado en sus trabajos construyendo diferentes modelos de clasificación para -a partir de la categoría-paraguas que suponen las "candidaturas independientes"- establecer una serie de subcategorías específicas que permitan ordenar fenómenos políticos heterogéneos en función de diferentes variables y objetivos de investigación.

Como consecuencia, la revisión de la literatura existente ofrece un amplio abanico de modelos teóricos construidos a partir de la noción general de "candidaturas independientes". Sin embargo, todos estos esquemas de definición se encuentran diseminados y disgregados a lo largo de las producciones académicas, no constando ejercicios de sistematización que pongan en diálogo los esfuerzos realizados por los autores arriba citados. Así las cosas, el primer objetivo de este trabajo es proponer un único esquema de categorización integral que conecte las contribuciones de los especialistas a la hora de definir diferentes realidades derivadas de la categoría general de "candidaturas independientes". Un ejercicio que se realizará en función de cuatro variables: (i) el origen de la "candidatura independiente"; (ii) la tipología de su electorado; (iii) la tipología del perfil militante y (iv) la tipología del líder de la "candidatura independiente".

En primer lugar, a partir de la categoría general de "candidaturas independientes" se pueden dividir tres subcategorías en función de su origen: de (i) si éstas provienen de movimientos sociales y/o asociaciones civiles (Holtmann, 2008; Ubasart, 2012); (ii) si son candidaturas herederas o bajo la influencia de formaciones partidistas tradicionales, esto es, "marcas blancas" (Holtmann, 2008; Ubasart, 2012; Reuchamps et al. 2012; Heyerick, 2016; Vampa, 2016) o bien si (iii) hunden sus raíces en proyectos autónomos, no descendiendo ni de movimientos sociales ni de los grandes partidos convencionales y/o estatales (Ehin et al., 2003; Ubasart, 2012; Reuchamps et al., 2012; Heyerick, 2016; Vampa, 2016; Ahedo y Ureta, 2017; Ureta, 2017);

Así, la primera de estas subtipologías en función del origen obedece a aquellas candidaturas provenientes de movimientos sociales y/o asociaciones civiles, estudiadas en los trabajos de Holtmann (2008) y Ubasart (2012). Mientras Hotmann (2008) no se detuvo en desarrollar excesivamente esta definición para el caso alemán; Ubasart (2012) ahondó teóricamente en los contenidos de esta subcategoría a la que 
denominó “candidaturas alternativas y populares". Éstas han irrumpido en la realidad catalana relativamente hace pocas legislaturas y hacen referencia a actores políticos locales que han sido objeto de procesos de institucionalización de los movimientos sociales y que presentan proyectos políticos que van más allá de la gestión de lo local, articulando luchas vecinales con reivindicaciones supramunicipales. La autora señaló que la subcategoría de "candidaturas alternativas y populares" presenta un nivel de politización alto: son formaciones con un modelo de municipio a defender a largo plazo, que implementan políticas públicas transformadoras, problematizan la gestión pública tradicional y son de base horizontal (Ubasart, 2011, 2012).

En segundo lugar, muchos autores han detectado en sus trabajos la existencia de una subtipología que tiene su origen en los partidos políticos convencionales y/o estatales: las"marcas blancas”. Esto es, formaciones que de forma implícita (moral) o explícita (financiera) cuentan con apoyo partidista, a pesar de no llevar aparejadas sus siglas (Ehin et al., 2003: 11; Holtmann, 2008; Ubasart, 2012; Reuchamps et al. 2012; Heyerick, 2016; Vampa, 2016; Ahedo y Ureta, 2017; Ureta, 2017). Este supuesto también puede contemplar coaliciones de partidos convencionales y/o estatales (Reuchamps et al., 2013; Heyerick, 2016; Vampa, 2016; Ahedo y Ureta, 2017; Ureta, 2017) así como escisiones (Ehin et al., 2003: 1; Ahedo y Ureta, 2017; Ureta, 2017).

Finalmente, dentro de la categoría general de "candidaturas independientes" desagregada en función de su origen también se encuentra la subtipología denominada "listas independientes". Se trata de una subtipología registrada por la mayoría de autores en sus respectivas investigaciones (Ehin et al., 2003; Ubasart, 2012; Reuchamps et al., 2012; Heyerick, 2016; Vampa, 2016; Ahedo y Ureta, 2017; Ureta, 2017). Todos ellos comparten en su definición el hecho de que se trata de formaciones no partidistas de personas independientes y autónomas con respecto de los partidos tradicionales/estatales. Algunos autores destacan además el trabajo de proximidad con la ciudadanía llevado a cabo por parte de estas candidaturas (Reuchamps et al., 2013), las cuales presentan un nivel de politización bajo, al margen de debates políticos amplios y transversales (Ubasart, 2011; 2012).

Asimismo, también se puede desagregar la categoría general de "candidaturas independientes" en subcategorías que respondan a la tipología del electorado, como hizo en su día Holtmann (2008) para diferenciar entre (i) candidaturas que representan el perfil social y los intereses de la vieja clase media (propietarios, artesanos y agricultores), donde priman los valores materialistas y (ii) candidaturas en defensa de la nueva clase media -sector servicios y funcionarios de alto rango- que se movilizan por preferencias de corte postmaterialista (Holtmann, 2008). Mientras, por su parte, la categoría general de "candidaturas independientes" también se puede desagregar en función de la variable "tipología del perfil militante", donde tanto Bracanti 
(2008) como González (2012) distinguieron entre: (i) candidaturas configuradas por miembros outsiders que no cuentan con experiencia política previa y (ii) candidaturas estructuradas por insiders que participaron previamente en funciones públicas (Brancanti, 2008; González, 2012).

Finalmente, la categoría general de "candidaturas independientes" se puede dividir en función de la tipología del perfil del líder de la candidatura (Ehin et al., 2013 Copus et al., 2008, 2009). Así, diversos autores (Copus et al., 2008; 2009) distinguieron (i) el completo independiente, aquel político que no es respaldado por ninguna formación y que -por tanto- no establece alianzas con otros independientes, siendo ésta "una elección individual y circunstancial" (Ehin et al., 2013: 11-12). En este sentido, Copus y sus colaboradores $(2008,2009)$ matizaron que el completo independiente no se reúne con políticos en espacios formales institucionalizados; aunque ello no signifique que esté completamente aislado, ya que interactúa de manera informal con los mismos. Por su parte, (ii) el independiente unido (cojoined) es aquel que forma parte de una coordinadora de independientes, donde la etiqueta "independiente" significa en este contexto "independencia" de un partido político. Mientras que -por último- (iii) el independiente revelado (revealed), es el candidato que coloca la etiqueta "independiente" en vez de las siglas del partido convencional, debido a disputas internas con la formación a nivel local o a una batalla por la reelección (Copus et al., 2008; 2009). A continuación se presenta un cuadro en el que se visualiza cómo se han estructurado las subcategorías de "candidaturas independientes":

\section{Cuadro 1}

Subtipologías de "candidaturas independientes"

\begin{tabular}{l|l}
\hline Variable & Definición \\
\hline Origen (procede de) & Movimientos sociales o agrupaciones civiles \\
& Partidos políticos convencionales y/o estatales \\
& Proyectos autónomos de personas independientes \\
\hline Tipología del electorado & Vieja clase media (materialismo) \\
& Nueva clase media (postmaterialismo) \\
\hline Tipología de perfil militante & Outsider \\
\hline Tipología de líderes & Insider \\
\hline & Completo independiente \\
& Independiente unido (cojoined) \\
\hline
\end{tabular}




\section{Nuevas posibilidades para su estudio dentro del ámbito municipal español}

A pesar de que los autores arriba mencionados han dedicado sendos esfuerzos a la hora de abordar la unidad de análisis de este estudio, lo cierto es que aún hay retos académicos de calado. En este sentido, las “candidaturas independientes" han sido tradicionalmente olvidadas en Ciencia Política; consideradas una categoría marginal y residual en la literatura existente (Ubasart, 2012; Ehin et al., 2013; Bautista, 2015; Maskarinec y Klimovsky, 2016; González, 2016). Ello se refleja en el hecho de que las producciones académicas han omitido de su agenda de interés científico el estudio de organizaciones políticas estrictamente locales, consideradas una anomalía política (González, 2012; 2016), outsiders (Brancanti, 2008: 650), una rareza queer (Ubasart, 2012), "el primo pobre" (Holtmann, 2008) o incluso los "parias" (Smith, 1991) de la politología. Ello se debe a que los estudios electorales y de partidos se han centrado mayoritariamente en las grandes formaciones políticas nacionales, dando la espalda de forma generalizada a hallazgos empíricos que señalan la importancia de la existencia de "candidaturas independientes" (Holtmann, 2008). Por tanto, se ha ignorado de forma amplia una singularidad extraordinaria del sistema (Boogers, 2008; Angenendt, 2016; González, 2016), un abandono científico que ha llevado a disponer de escasa información cualitativa previa sobre el objeto de estudio.

Si se aterriza en el ámbito municipal español, a pesar de que algunos trabajos han abordado esta realidad (Botella, 1990; Capo, 1991; Delgado, 1997), pocos han profundizado en el fenómeno (Ubasart, 2012; Bautista, 2015, Ahedo y Ureta, 2017; Ureta, 2017), ya que la mayoría de los focos de atención se han aglutinado en torno a los grandes partidos políticos que operan a nivel tanto estatal como autonómico, dejando en un segundo plano el estudio de partidos locales, especialmente de "candidaturas independientes". Ello no resulta extraño si se tiene en cuenta que los partidos convencionales se ocuparon de garantizar su preeminencia a nivel municipal en España cuando regularon su funcionamiento en la esfera local a través de la Ley de Elecciones Locales de 1978. "El objetivo primordial perseguido por esta norma era consagrar las formaciones políticas como protagonistas del proceso político local" (Botella, 1992: 150), siendo "el temor de los principales partidos el surgimiento de ofertas puramente locales" (Botella, 1992: 150). Sin embargo -desde aquellos años- en España se han experimentado muchos cambios conducentes a superar, transformar y contestar el rol excesivamente dominante y gerencialista de los partidos tanto convencionales como nacionales o regionales en la esfera municipal, lo que supone un nuevo marco que abre posibilidades de estudio en torno a actores políticos locales madrugadores como las "candidaturas independientes".

Es por ello que el segundo objetivo de este trabajo es presentar evidencias empíricas a partir de las cuales se fundamentan las nuevas posibilidades de investigar las 
"candidaturas independientes" en la esfera municipal española. Así, estudios muy recientes (Kening y Rahat, 2018) desvelan que efectivamente esas posibilidades de investigación a las que se aludía tienen una base empírica apoyada en sólidos datos. Según Kening y Rahat (2018), mientras en 1985 el porcentaje de votos logrados por "candidaturas independientes" en los Ayuntamientos españoles apenas alcanzaba el $7,4 \%$, este porcentaje ha ido incrementándose legislatura tras legislatura hasta que en 2015 -año de las últimas Elecciones Municipales españolas- el porcentaje de voto obtenido por la categoría general de "candidaturas independientes" asciende a 15,4\%, lo cual supone un aumento del $8 \%$ en términos de votos alcanzados. Por tanto, se va consolidando una tendencia positiva en el crecimiento electoral de las "candidaturas independientes" en el ámbito municipal español; el cual se erige como territorio prometedor en donde la unidad de análisis emerge y crece exponencialmente, logrando mayor porcentaje de votos y consolidándose así como un actor político cada día más relevante en términos cuantitativos.

En el siguiente cuadro se muestran los datos ofrecidos en el último estudio de Kening y Rahat (2018):

\section{Cuadro 2}

Evolución de votos (\%) de "candidaturas independientes" (1985-2015)

en el ámbito municipal español

\begin{tabular}{l|c|c|c|c|c|c|c}
\hline País & 1975 & 1985 & 1995 & 2005 & 1995 & 2015 & Tendencia \\
\hline \multicolumn{8}{c}{ Evolución de votos (\%) de “candidaturas independientes” locales en España } \\
\hline Escaños & - & 7,4 & 3,3 & 6,6 & 232 & 15,4 & + \\
\hline
\end{tabular}

Elaboración propia a partir de Kening y Rahat (2018)

Este progresivo aumento en votos no ha pasado desapercibido a nivel internacional, pues Kenin y Rahat (2018) señalan que el crecimiento más significativo de "candidaturas independientes" operativas a nivel municipal en Europa se concentra precisamente en España, Portugal, Holanda, Italia e Irlanda ${ }^{1}$. Estos datos confirman los resultados de la investigación de Bautista (2015), quien realizó un censo de "candidaturas independientes" locales en Espala entre 2011 y 2015. A juicio de Bautista (2015), la evidencia empírica de sus estudios revela la "aparición de numerosas

1. Sin embargo, hay que tener en cuenta que existen diferencias importantes entre una candidatura independiente española y las estudiadas a nivel internacional, debido a sistemas electorales y sistemas de partidos distintos que influyen en las condiciones de su evolución. 
opciones políticas que se presentan como alternativa a los partidos tradicionales y -a merced de sus buenos resultados electorales- están originando un cambio en el mapa político municipal” (Bautista, 2015: 19). En este sentido, Bautista ofrece datos sumamente interesantes - desgranados en función de las provincias españolas- a partir de los cuales se abren posibilidades para emprender estudios cualitativos más profundos que permitan conocer mejor las diferentes realidades del fenómeno de "candidaturas independientes" en distintas Comunidades Autónomas españolas.

De hecho, las regiones catalana y vasca ya han sido delimitaciones territoriales escogidas para investigar las "candidaturas independientes". Tanto Ubasart (2011, 2012) como Ahedo y Ureta (2017) identificaron un creciente incremento de la unidad de análisis en estas delimitaciones, y mientras Ubasart $(2011,2012)$ se centró en la subcategoría de "candidaturas alternativas y populares" para conocer el perfil de sus militantes; Ahedo y Ureta (2017) focalizaron su atención en la tipología de actor político que las "listas locales" representan en el País Vasco. Sin embargo -y a pesar de estos trabajos reseñados- las "candidaturas independientes" apenas han sido estudiadas en profundidad en el nivel municipal español; un aspecto que llama la atención si se tiene en consideración la relevancia que toma esta unidad de análisis en términos cuantitativos (Bautista, 2015; Kening y Rahat, 2018).

Es por ello que este trabajo insiste en las nuevas posibilidades de estudio que brotan a partir de evidencias empíricas, las cuales justifican el abordaje de las "candidaturas independientes" locales como objeto de estudio en España. Existen interesantes preguntas de investigación en torno a este actor, que podrían responder a "cómo se forman estos órganos políticos, cuáles son sus singularidades, particularidades, similitudes y diferencias entre sí, las condiciones y factores que inciden en su permanencia o desaparición del sistema político" (González, 2016: 125). Por ello, hay una necesidad imperiosa de contestar a las preguntas de investigación que plantean estos fenómenos, bien profundizando en estudios de caso o aplicando el método comparativo para poner en diálogo las "candidaturas independientes" que operan en delimitaciones territoriales distintas.

\section{Factores explicativos del surgimiento y crecimiento electoral de "candidatu- ras independientes"}

El tercer objetivo de este estudio persigue conocer los factores estructurales generales a partir de los cuales se puede comenzar a explicar por qué surgen y aumentan en votos las "candidaturas independientes". Un trabajo que futuros investigadores deberán complementar -además- con aquellos rasgos específicos que expliquen la irrupción y progresión de "candidaturas independientes" en las respectivas delimitaciones territoriales que escojan. Así las cosas, se destacan cuatro elementos estructurales 
generales a partir de los cuales se puede comenzar a interpretar el contexto en el que las "candidaturas independientes" nacen y crecen electoralmente a nivel municipal en España: (i) crisis de la democracia representativa (ii) crisis de los grandes partidos políticos convencionales-estatales (iii) amplia deslegitimación de los representantes políticos y (iv) creciente importancia y reforzamiento del ámbito municipal en España-nuevo municipalismo-.

En primer lugar, se está produciendo una creciente deslegitimación del sistema de democracia liberal (Subirats, 2001; Jessop, 2008: 219) que algunos autores han denominado "malestar con la democracia" (Norris, 1999; Smith, 2009; Geissel, 2013), insatisfacción (Mény, 2003), desilusión (Hindess, 2010) desencantamientos, males, desmitificación o desconsolidación (Dalton y Shin, 2006; Habermas, 1973); una lógica de regresión democrática (Gomá y Blanco, 2016: 26) e incluso privatización de la misma (Goikoetxea, 2017) donde se comienza a poner en duda "la sostenibilidad de la democracia en momentos de cambio acelerado (...) Y más cuando se constata un déficit de eficacia de sus mecanismos decisorios ante problemas cada vez más complejos y enquistados" (Montero et al., 2006: 27; Subirats, 2001: 34).

Esa desconfianza hacia el sistema de democracia liberal se materializa en una desafección hacia las instituciones de representación del Estado (Subirats, 2001; Jessop, 2008). Por eso, en segundo lugar, se está produciendo hoy en día un desapego con respecto a los grandes partidos (Jessop, 2008: 218) generadores de una creciente desafección política detectada en la mayoría de estudios (Papadopoulos, 2015), donde la erosión de las formaciones políticas convencionales en su papel de mediadores con la sociedad es tan profunda que muchas organizaciones políticas han perdido su duradero monopolio (Kening y Rahat, 2018). Los grandes partidos políticos ya no son capaces de completar su función representativa y legitimadora (Webb, 1995; Whiteley y Seycl, 1998; Dalton y Wattenberg, 2000; Mair y Van Biezen, 2001; Heyerick, 2016), donde las formaciones políticas profesionalizadas experimentan un declive en la afiliación e identificación partidista, así como una ascendente desconfianza (Owen y Dennis, 1996; Norris, 1999; Wattenberg, 2000; Mair y Van Biezen, 2001; Dalton, 2004; Berglund et al., 2005; Holmberg, 2007; Dalton y Welzel, 2014), por lo que si estas tendencias prueban a ser sistémicas, los partidos clásicos pueden perder su estatus como vehículos de la representación al no ser considerados como los mejores instrumentos para alcanzar objetivos políticos (Boeyer y Weeks, 2009). Es en este contexto de profunda crisis partidista donde las "candidaturas independientes" emergen y crecen en nivel de representatividad.

Así es como se puede entender que en muchos países "los partidos nacionales hayan perdido su duradero monopolio en la política local a favor de las candidaturas independientes" (Kening y Rahat, 2018). En este sentido, distintos trabajos señalan a 
las mismas como "alternativa a los partidos nacionales crecientemente rechazados" (Reuchamps et al., 2017) debido a que las comunidades locales se han visto obligadas a imaginar fórmulas diferentes (González, 2016: 145) frente a las carencias organizativas (Mair, 2005; Copus et al., 2009) y fallos de representación de la tradicional política local (Mair, 2005; Copus et al., 2009; Holtmann et al., 2011: 20; Copus et al., 2013: 6; Radzik-Maruszak, 2016).

En tercer lugar, se está produciendo una amplia deslegitimación de los representantes políticos. Por un lado, la mayoría de ellos son considerados profesionales de la política (Wattenberg, 2000; Mair y Van Biezen, 2001; Holmberg, 2007; Dalton y Welzel, 2014), lo que implica que se les percibe como gestores dentro de un contexto donde la cultura del New Management, la impersonalidad de las tecnologías, (Giddens, 2000: 23), la burocratización de los procedimientos y la técnica se imponen como ideología dominante (Habermas, 1968). A ello hay que añadir los escándalos de corrupción y las acusaciones de incompetencia y traición que han perseguido a diferentes representantes políticos y que han generado resentimiento contra las élites gobernantes (Antón y Hernández, 2016). De nuevo, la literatura de referencia encuentra en los procesos de desinstitucionalización del sistema de partidos el problema recurrente de la corrupción política por parte de los representantes (Dietz y Myers, 2007), lo cual desencadena una pérdida de credibilidad en los electores que ven la corrupción como causante del deficiente funcionamiento del sistema democrático, las instituciones públicas y los partidos políticos (Lupu, 2014: 564). Un escenario donde las candidaturas independientes han encontrado el terreno de su éxito electoral.

En cuarto y último lugar, cabe resaltar que el auge y progresión de las "candidaturas independientes" se está produciendo en un contexto de recuperación y reforzamiento de la arena local y de la figura del Ayuntamiento, una revitalización que se explica a través de las siguientes tesis que funcionan como procesos: (i) la tesis de la politización y (ii) del empoderamiento local, así como en la del (iii) nuevo localismo que otorgan sentido al fenómeno de reactivación del campo municipal y que -por tanto- ofrecen herramientas analíticas para entender asimismo el fenómeno de las "candidaturas independientes" en España. En primer lugar, los procesos de politización local se guían por una democracia más participativa (Brugué y Gomá, 1998: 21-22), generándose una cultura política crítica. (ii) La creciente importancia del ámbito municipal también se basa en el empoderamiento de muchos ayuntamientos, que hoy por hoy rechazan visiones gerencialistas y adoptan "nuevos roles estratégicos cualitativos" (Blanco y Gomá, 2002: 23) mediante el "desbordamiento de las agendas locales que a menudo han ido más allá de sus bases competenciales" (Blanco y Gomá, 2002) y de sus límites presupuestarios. Finalmente, (iii) la importancia del territorio 
local se debe a que (iii) han aparecido nuevos "actores locales en espacios donde se deciden e implementan las políticas públicas" (Brugué y Gomá, 1998: 23), como es el caso de las "candidaturas independientes" en España.

Estas tendencias y cambios -así como los resultados electorales que revelan una profunda necesidad de estudiar las candidaturas independientes en España- se refuerzan si se contrastan y entrecruzan con los datos de la valoración positiva que la ciudadanía otorga al Ayuntamiento, aquella institución mejor situada para conocer y dar respuesta a las demandas y necesidades de ciudadanos desde la proximidad (Brugué y Gomá, 1998: 19), especialmente en momentos de agudización de la crisis multidimensional que requiere también de respuestas locales que reterritorialicen problemas globales ${ }^{2}$. Por ello, es necesario prestar más atención al nivel municipal y -específicamente- focalizar la concentración en las "candidaturas independientes", las cuales cada día ganan más votos en la esfera local.

\section{Bibliografía}

AHEDO, Igor y Miriam URETA (2017) "Kuasi-mugimendutze prozesu baterantz? Bizkaian gobernatzen duten "herri plataforma independienteak", Inguruak, 63, 51-69.

ANGENENDT, Michael (2016) "Anti-Party Sentiment among Members in German Local Parties. Concpetual Framework and Empirical Analysis”, ECPR Graduate Students Conference, 10-13, July 2016, Tartu.

ANTÓN, Joan y Aitor HERNÁNDEZ (2016) "El crecimiento electoral de la derecha radical populista en Europa: parámetros ideológicos y motivaciones sociales", Política y Sociedad, 53 (1), 17-28.

BAUTISTA, Juan (2015) "Los partidos del ámbito local en España. Estudio cuantitativo de la legislatura 2011-2015”, XII Congreso AECPA ¿Dónde está hoy el poder?, 13-15, Julio de 2015.

BERGLUND, Frode, Sören HOLMBERG, Hermann SCHMITT and Jacques THOMASSEN (2005) "Party identification and party choice", in Jaques THOMASSEN (ed.) The European Voter, Oxford: Oxford University Press, 106124.

BLANCO, Ismael y Ricard GOMÁ (2002) "Proximidad y participación: marco conceptual y presentación de experiencias”, en Ismael BLANCO y Ricard GOMÁ (coords.) Gobiernos locales y redes participativas. Barcelona: Ariel, 21-42.

2. En este sentido, a la luz de los resultados que pone de manifiesto los Barómentros del CIS entre 1994 y 2008 en su apartado "escala de confianza en las instituciones", los ayuntamientos siempre han sido mejor valorados que las instituciones de niveles autonómicos y nacionales. 
BOOGERS, Marcel and Gerrit VOERMAN (2010) "Independent Local Political Parties in the Netherlands", Local Government Studies, 36(1), 75-90. https://doi. org/10.1080/03003930903435807.

BOOGERS, Marcel (2008) "Local Political Parties in the Netherlands: Anomaly or Prototype?" REISER, in Marion and Everhard HOLTMANN (eds.) Farewell to the Party Model? Independent Local Lists in East and West European Countries, Manchester: VS Verlag, 149-167.

BOTELLA, Joan (1990) "Les élites polítiques de la Catalunya democrática”, Papers de Sociología, (33), 66-74. https://doi.org/10.5565/rev/papers/v33n0.1550.

BOTELLA, Joan (1992) "La galaxia local en el sistema político español”, Revista de Estudios Políticos, (76), 145-160.

BRACANTI, Dawn (2008) "Winning Alone: The Electoral Fate of Independent Candidates Worldwide", The Journal of Politics, 70(3), 648-662. https://doi. org/10.1017/s0022381608080675.

BRUGUÉ, Quim y Ricard GOMÁ (1998) "Las políticas locales: agendas complejas, roles estratégicos y estilo relacional”, en Quim BRUGUÉ y Ricard GOMÁ (coords.) Gobiernos locales y políticas públicas, Barcelona: Ariel, 25-38.

CAPO, Jordi (1991) “Elecciones municipales, pero no locales”, Revista Española de Investigaciones Sociológicas, 56, 143-164. https://doi.org/10.2307/40199497.

COPUS, Colin, Clark ALISTAIR, Reynaert, HERWIG and Kristof STEYVERS (2009) "Minor party and independent politics beyond the mainstream: fluctuating fortunes but a permanent presence", Parliamentary Affairs, 1, 4-18. https://doi. org/10.1093/pa/gsn035.

COPUS, Colin, Clark ALISTAIR and Karin BOTTOM (2008) "Multi-Party Politics in England? Small Parties, Independents and Political Associations in English Local Politics", in Marion REISER and Everhand HOLTMANN (eds.) Farewell to the party Model? Independent Local Lists in Eastern and Western European Countries, Wiesbaden: VS Verlag, 253- 76.

COPUS, Colin and Gissur, ERLINGSSON (2012) "Parties in local government: A review”, Representation, 48(2), 235-247. https://doi.org/10.1080/00344893.2012 .683489 .

COPUS, Colin and Gissur ERLINGSSON (2013) "Formal institutions versus informal decision-making. On parties, delegation and accountability in local government", Scandinavian Journal of Public Administration, 17(1), 51-68.

DALTON, Rusell (2003) Democratic Challenges, Democratic Choices: The Erosion of Political Support in Advanced Industrial Democracies, Oxford: University Press.

DALTON, Russell; SHIN, Chull (2006): “Citizens, Democracy and Markets" in Russell DALTON and Doh Chull SHIN (eds.) Citizens, Democracy and Markets around the Pacific Rim, Oxford: Oxford University Press. 
DALTON, Rusell and Martin WATTENBERG (2000) "Partisan Change and the Democratic Process", in Rusell DALTON and Martin WATTENBERG (eds.) Parties without Partisans: Political Change in Advanced Industrial Democracies, Oxford: Oxford University Press, 261-284.

DALTON, Rusell and Christian WELZEL (2014) The Civic Culture Transformed. From Allegiant to Assertive Citizens, Cambridge: Cambridge University Press. https://doi.org/10.1017/CBO9781139600002.

DELGADO, Irene (1999) "Resultados electorales y orientación del voto en los comicios municipales de 1995”, Revista Española de Investigaciones Sociológicas, 86, 247-273. https://doi.org/10.2307/40184152.

EHIN, Piret, Madise ÜLLE, Mihkel SOLVAK, Rein TAAGEPERA, Vassil, KRISTJAN and Vinkel PRIIT (2013) "Independent candidates in national and European elections”, Brussels: European Parliament. http://www.europarl.europa.eu/ thinktank/en/document.html?reference=IPOL-AFCO_ET(2013)493008.

GEISSEL, Brigitte (2013) "On the Evaluation of Participatory Innovations- A Preliminary Framework" in Brigitte GEISSEL and Marko JOAS (eds.) Participatory Democratic Innovations in Europe. Improving the Quality of Democracy?, Berlin and Toronto: Barbara Budrich Publishers.

GIDDENS, Anthony (2000) Un mundo desbocado. Madrid: Editorial Alfaguara.

GOIKOETXEA, Jule (2017): Privatizing Democracy. Global Ideals, European Politics and Basque Territories, Berlín: Peter Lang.

GONZÁLEZ, Roy (2012): "Vuelta a lo local: un enfoque teórico-metodológico para el análisis de partidos políticos subnacionales", 22nd World Congress of Political Science, 8-12, Julio 2016, Madrid.

GONZÁLEZ, Roy (2016) "La difícil construcción de la democracia desde abajo: apuntes sobre la inexperiencia de partidos políticos locales en el Estado de Méjico", Revista de Temas Contemporáneos sobre Lugares, Política y Cultura, (6)2, 125-152.

HABERMAS, Jürgen (1968) Ciencia y técnica como ideología, Madrid: Tecnós.

HABERMAS, Jürgen (1973) Problemas de legitimación y capitalismo tardío, Buenos Aires: Amorrortu.

HEYERICK, An (2016) "The organized strength of non-national lists in Flanders", ASSP Congress, 20-21, January 2016, Belgium.

HINDESS, Barry (2010) "Men Behaving Badly" Revue Internationale de Philosiphie, 251(64), 39-57.

HOLTMANN, Everhart (2008) "Local Governance and Party Systems Change: A Cross- National Perspective", IPSA Conference on International Political Science, 30-2, April and May, Quebec. 
HOLMBERG, Sören (2007) Partisanship Reconsidered. The Oxford Handbook of Political Behaviour, Oxford: Oxford University Press.

JESSOP, Robert (2008) El futuro del Estado capitalista, Madrid: Catarata.

Lupu, Noam (2014) "Brand Dilution and the Breakdown of Political Parties in Latin America", World Politics, 66 (4), 561-602.

MAIR, Peter (2005) Democracy beyond parties, Irvine: Center for the Study of Democracy.

MAIR, Peter and Ingrid VAN BIEZEN (2001) "Party Membership in Twenty European Democracies, 1980-2000”, Party Politics, (7)1, 5-21.

MASKARINEC, Pavel and Daniel KLIMOVSKY (2016) "Independent Candidates in the Local Elections of 2014 in the Czech Republic and Slovakia", Lex Localis, (14)4, 853-871. https://doi.org/10.4335/14.3.511-520(2016).

MÉNY, Yves (2003): "De la Démocratie en Europe: Old Concepts and New Challenges", Journal of Common Market Studies, 41(1), 1-13.

MONTERO, José Ramón, Joan FONT y Mariano TORCAL (2006) “Ciudadanos, asociaciones y activistas”, en MONTERO, José Ramón, José FONT y Mariano TORCAL (eds.) Ciudadanos, asociaciones y participación en España.. Madrid: CIS, 25-46

NORRIS, Pippa (1999) Critical Citizens. Global Support for Democratic Governance, Oxford: Oxford University Press.

PAPADOPOUlOS, Yannis (2013) Democracy in Crisis? Politics, Governance and Policy, Basingstoke: Palgrave McMillan.

RAHAT, Gideon and Ofer KENING (2018) From Party Politics to Personalized Politics? Party Change and Political Personalization in Democracies, Oxford: Oxford University Press.

REUCHAMPS, Min, Vincent JACQUET, Jérémy DODEIGNE Régis DANDOY and Geoffroy MATAGNE (2013) "Gimme your name and I'll tell you who you are. An exploration of the name of local lists", Annual Conference of Elections, Public Opinion and Parties (EPOP), 13-15, September 2016, Lancaster.

SMITH, Graham (2009): Democratic Innovations. Designing Institutions for Citizen Participation, Cambridge: Cambridge University Press.

SUBIRATS, Joan (2001) "Nuevos mecanismos participativos y democracia: promesas y amenazas", en FONT, Joan (coord.) Ciudadanos y decisiones públicas, Barcelona: Ariel, 33-42

UBASART, Gemma (2012) Candidatures alternatives $i$ populars a Catalunya: construint democracia. Barcelona: Icaria. 
UBASART, Gemma (2012) "Municipalismo alternativo y popular ¿Hacia una consolidación de las tesis del nuevo localismo y la politización del mundo local?", Revista de Estudios Políticos, 157, 135-162.

URETA, Miriam (2017) "La emergencia de las plataformas vecinales independientes que gobiernan en Bizkaia: un híbrido entre movimiento social y partido político como alternativa", Encrucijadas Revista Crítica de Ciencias Sociales, 13, 4-21.

VAMPA, Davide (2016) “Declining partisan representation at the local level: assessing and explaining the strengthening of local lists in Italian municipalities (19952014)", Local Government Studies, 44(2), 579-597. https://doi.org/10.1080/0300 3930.2016.1146137.

WATTENBERG, Martin (2000) “The Decline of Party Mobilization", in WATTENBERG, Martin and Rusell DALTON (eds.) Parties without Partisans: Political Change in Advanced Industrial Democracies, Oxford: Oxford University Press.

WEBB, Paul (1995) “Are British Political Parties in Decline?", Party Politics, 1(3) 299322. https://doi.org/10.1177/1354068895001003001.

WHITELEY, Paul and Patrick SEYD (1998) “The Dynamics of Party Activism in Britain: A Spiral of Demobilisation”, British Journal of Political Science, 28, 113138. https://doi.org/10.1017/s000712349800012x. 
Herramientas teóricas para la definición y análisis de las "candidaturas independientes" locales: nuevas posibilidades de estudio en el ámbito municipal español Miriam Ureta García 
Herramientas teóricas para la definición y análisis de las "candidaturas independientes" locales: nuevas posibilidades de estudio en el ámbito municipal español Miriam Ureta García 
